\title{
$\mathrm{rGO} / \mathrm{SiC}$ 复合材料的制备与性能研究
}

\author{
黄毅华 ${ }^{1}$, 江东亮 ${ }^{1,2}$, 陈忠明 ${ }^{1}$, 刘学建 ${ }^{1}$, 张先锋 ${ }^{3}$, 廖振鬼 ${ }^{4}$, 黄政仁 ${ }^{1,2}$
}

(1. 中国科学院 上海硅酸盐研究所, 结构陶瓷与复合材料工程研究中心, 上海 200050; 2. 中国科学院 上海硅酸盐 研究所, 高性能陶瓷和超微结构国家重点实验室, 上海 200050; 3. 南京理工大学, 南京 210094; 4. 上海浩魁材料 科技有限公司, 上海 200444)

摘 要: 碳化硅 $(\mathrm{SiC})$ 陶瓷具有优异的力学性能, 但是其断裂韧性相对较低。石墨烯的引入有望解决碳化硅陶瓷的断 裂韧性较低的问题。本研究采用热压烧结工艺, 制备了具有不同还原-氧化石墨烯(rGO)掺入量的 SiC 复合材料。经 过 $2050^{\circ} \mathrm{C}$ 保温、 $40 \mathrm{MPa}$ 保压 $1 \mathrm{~h}$ 后, 所制备的复合材料均烧结致密。对复合材料中 rGO 的掺入量、微观结构和力 学性能的相互关系进行分析和讨论。加入 $4 \mathrm{wt} \%$ 的 rGO 后, 复合材料的三点抗弯强度达到 $564 \mathrm{MPa}$, 比热压 SiC 陶 瓷提高了 $6 \%$; 断裂韧性达到 $4.02 \mathrm{MPa} \cdot \mathrm{m}^{1 / 2}$, 比热压 $\mathrm{SiC}$ 陶瓷提高了 $54 \%$ 。加入 $6 \mathrm{wt} \%$ 的 $\mathrm{rGO}$ 后, 复合材料的三点 抗弯强度达到 $420 \mathrm{MPa}$ ，略低于热压 $\mathrm{SiC}$ 陶瓷，但其断裂韧性达到 $4.56 \mathrm{MPa} \cdot \mathrm{m}^{1 / 2}$, 比热压 $\mathrm{SiC}$ 陶瓷提高了 $75 \%$ 。裂 纹扩展微观结果显示, 主要增韧机理有裂纹偏转、裂纹桥连和 rGO 片的拔出。

关 键 词: 石墨烯; 碳化硅陶瓷; 定向掺杂

中图分类号: TQ174 文献标识码: A

\section{Fabrication and Property of rGO/SiC Composite}

\author{
HUANG Yi-Hua ${ }^{1}$, JIANG Dong-Liang ${ }^{1,2}$, CHEN Zhong-Ming ${ }^{1}$, LIU Xue-Jian ${ }^{1}$, \\ ZHANG Xian-Feng ${ }^{3}$, LIAO Zhen-Kui ${ }^{4}$, HUANG Zheng-Ren ${ }^{1,2}$
}

(1. Structural Ceramics and Composites Engineering Research Center, Shanghai Institute of Ceramics, Chinese Academy of Sciences, Shanghai 200050, China; 2. State Key Laboratory of High Performance Ceramics and Superfine Microstructure, Shanghai Institute of Ceramics, Chinese Academy of Sciences, Shanghai 200050, China; 3. Nanjing University of Science and Technology, Nanjing 210094, China; 4. Shanghai Haokui Material Ltd, Shanghai 200444, China)

Abstract: SiC ceramics have excellent mechanical properties, but its toughness is relatively low. To enhance the fracture toughness of $\mathrm{SiC}$ ceramics, graphene is introduced as fillers. In this study, the silicon carbide-reduced graphene oxide ( $\mathrm{SiC} / \mathrm{rGO}$ ) composites with different contents of rGO were fabricated by hot press sintering (HP). Near fully-dense SiC/rGO composite was obtained after being hot-pressed under $2050^{\circ} \mathrm{C}, 40 \mathrm{MPa}$ for $1 \mathrm{~h}$. In addition, the influences of graphene reinforcement on the sintering process, microstructure, and mechanical properties (fracture toughness, bending strength, and Vickers hardness) of SiC/rGO composites were discussed. The three-point flexural strength of $4 \mathrm{wt} \% \mathrm{rGO} / \mathrm{SiC}$ composite reached $564 \mathrm{MPa}$, and the fracture toughness reached $4.02 \mathrm{MPa} \cdot \mathrm{m}^{1 / 2}$, which were $6 \%$ and $54 \%$ higher than those of hot-pressed $\mathrm{SiC}$ ceramics, respectively. The flexural strength of the three points of $6 \mathrm{wt} \% \mathrm{rGO} / \mathrm{SiC}$ composite was $420 \mathrm{MPa}$, which was lower than that of hot-pressed SiC ceramics. While its fracture toughness was up to $4.56 \mathrm{MPa} \cdot \mathrm{m}^{1 / 2}$, which was $75 \%$ higher than that of hot-pressed $\mathrm{SiC}$ ceramics. The results of crack propagation show that the toughening mechanism can be ascribed to crack deflection, crack bridging and rGO pullout.

Key words: reduced graphene oxide (rGo); SiC ceramics; oriental doping

收稿日期：2018-02-10; 收到修改稿日期：2018-06-08

基金项目：国家自然科学基金(51572276); 中国科学院青年创新促进会项目

National Natural Science Foundation of China (51572276); Youth Innovation Promotion Association, Chinese Academy of Sciences

作者简介：黄毅华(1982-), 男, 副研究员. E-mail: wyu@mail.sic.ac.cn

通讯作者：黄政仁, 研究员. E-mail: zhrhuang@mail.sic.ac.cn 
石墨烯具有优异的性能, 是目前已知强度最高 的物质 ${ }^{[1-4]}$ 。碳化硅 $(\mathrm{SiC})$ 是传统的结构-功能陶瓷 ${ }^{[5]}$, 具有优良的力学、热学、电学等特性, 被广泛应用 于防弹装甲、精密轴承、热交换器部件等领域 ${ }^{[6-7]}$ 。 但是, 碳化硅的韧性较差, 限制了其优异性能的发 挥。碳化硅是制备高质量、大尺寸石墨烯的最佳原 料之一，与石墨烯有很好的相容性 ${ }^{[8]}$ 。石墨烯/碳 化硅陶瓷的研究也是近年来无机材料领域的一个热 点 ${ }^{[9-12]}$ 。Belmonte 等 ${ }^{[13]}$ 利用 SPS 烧结技术(液相, 添 加剂 5wt $\% \mathrm{Y}_{2} \mathrm{O}_{3} 、 3 \mathrm{wt} \% \mathrm{Al}_{2} \mathrm{O}_{3}$ )制备并比较了石墨烯 纳米片(10 20 nm 厚, $14 \mu \mathrm{m}$ 面向尺寸)、还原-氧化 石墨烯(5nm 厚, $5 \mu \mathrm{m}$ 面向尺寸)对石墨烯/碳化硅复 合材料静态力学性能的影响。当掺入含量均为 $5 \mathrm{vol} \%$ 时, 加入石墨烯纳米片的复合材料抗弯强度 达到 $622 \mathrm{MPa}$, 而加入还原一氧化石墨烯的复合材料 抗弯强度略低, 但均比未加入石墨烯的提高了 60\% 70\%; 加入 $5 \mathrm{vol} \%$ 还原-氧化石墨烯的复合材 料的断裂韧性 $\left(K_{\mathrm{IC}}\right)$ 高达 $8.3 \mathrm{MPa} \cdot \mathrm{m}^{1 / 2}$, 比未加入石 墨烯的提高了 $162 \%$ 。石墨烯纳米片含量对复合材 料的静态力学性能有影响, 加入量为 $10 \mathrm{vol} \%$ 时, 其 断裂韧性最高, 在 $5.7 \mathrm{MPa} \cdot \mathrm{m}^{1 / 2}$ 左右。电镜分析结果 显示, 石墨烯/碳化硅陶瓷的静态力学性能的提高是 由于石墨烯片层对碳化硅晶粒的桥连作用。此外, 他们利用少层石墨烯在碳化硅上的自生长, 获得了 $4 \mathrm{vol} \%$ 石墨烯掺杂的复合材料，其断裂韧性为 $4.5 \mathrm{MPa} \cdot \mathrm{m}^{1 / 2}$, 维式硬度为 $18.6 \mathrm{GPa}^{[14]}$ 。张玉军等 ${ }^{[15]}$ 采用无压固相烧结法制备了石墨烯(3 nm 厚)/碳化 硅复合材料。掺入 $1 \mathrm{wt} \%$ 石墨烯后, 无压烧结的碳化 硅陶瓷复合材料的断裂韧性(单边开槽法 SENB)达 到 $5.65 \mathrm{MPa} \cdot \mathrm{m}^{1 / 2}$, 比未加入石墨烯的提高了 $22.6 \%$; 三点抗弯强度为 $434.14 \mathrm{MPa}$, 维式硬度为 $29.21 \mathrm{GPa}$ 。

相比 SPS 方法，热压烧结可以制备尺寸更大的 样品。Richard 等 ${ }^{[16]}$ 首次采用热压烧结法制备了石墨 烯纳米片/碳化硅复合材料, 但是由于采用石墨烯作 为烧结助剂, 导致晶粒过度生长, 部分晶粒尺寸达 到 $20 \mu \mathrm{m}$, 材料抗弯强度只有 $290 \mathrm{MPa}$ 。本工作采用 热压烧结法, 采用嗍一碳作为烧结助剂, 制备具有石 墨烯纳米片定向掺杂的碳化硅陶瓷复合材料, 以期 获得更好的力学性能。

\section{1 实验方法}

实验原料选用平均粒径为 $0.5 \mu \mathrm{m}$ 的 $\mathrm{SiC}$ 粉体 (FCP-1, Norton Co, Lillesand, Norway), 还原-氧化 石墨烯(常州第六元素)作为掺入相, 碳化硼 $\left(\mathrm{B}_{4} \mathrm{C}\right)$ 和
无定形碳(C)作为烧结助剂。将上述原料 $\mathrm{SiC}$ 粉体, $\mathrm{B}_{4} \mathrm{C}-0.5 \mathrm{wt} \%$ (占粉体总量), C- $1 \mathrm{wt} \%$ 与 $0 、 1 \mathrm{wt} \%$ 、 $2 \mathrm{wt} \% 、 4 \mathrm{wt} \% 、 6 \mathrm{wt} \%$ 的 rGO 按照比例加入到无水乙 醇中, 原料球磨混合后干燥, 并过篎后在热压烧结 炉中经 $2050^{\circ} \mathrm{C} 、 50 \mathrm{MPa}$ 保温 $1 \mathrm{~h}$ 烧结。

采用阿基米德法测定烧结后 $\mathrm{rGO} / \mathrm{SiC}$ 陶瓷复 合材料的密度。采用三点弯曲法在 Instron 5566 材 料试验机测试 $\mathrm{rGO} / \mathrm{SiC}$ 陶瓷复合材料的力学性 能, 样品尺寸为 $3 \mathrm{~mm} \times 4 \mathrm{~mm} \times 36 \mathrm{~mm}$, 加载速率为 $0.5 \mathrm{~mm} / \mathrm{min}$, 性能数据为 5 根试样数据的平均值。 采用维氏硬度压痕法来测试复合材料的断裂㓞性, 性能数据为 5 个压痕裂纹韧性的平均值。采用扫描 电镜(Scanning Electron Microscopy, SEM)观察 SiC 陶瓷复合材料的微观结构; 采用透射电镜(TEM)观 察 $\mathrm{rGO}$ 和 $\mathrm{rGO} / \mathrm{SiC}$ 陶瓷复合材料离子减薄片的微 观结构。在 SEM 照片中随机取 200 个晶粒尺寸的平 均值, 得到复合材料中碳化硅的晶粒尺寸。

\section{2 结果与讨论}

\section{1 还原一氧化石墨烯性能}

通常将层数少于 10 层的石墨二维材料统称为 石墨烯。本研究采用的还原一氧化石墨烯( $\mathrm{rGO}$ )微观 形貌如图 1 所示, 层间距离为 $0.331 \mathrm{~nm}$, 是典型的 石墨(002)面的面间距离。其中, 层数最少为 5 层, 一 般为几十层至上百层, 厚度小于 $100 \mathrm{~nm}$ 。因此, 本 研究所用石墨烯材料为石墨烯纳米片范畴, 并非严 格意义上的少层石墨烯。

图 2 是 $\mathrm{rGO}$ 在 $2050^{\circ} \mathrm{C}$ 煅烧前后的 SEM 照片。 经过高温㷽烧, $\mathrm{rGO}$ 并未发生分解, 面向尺寸为 $8 \mu \mathrm{m}$ 左右。轱烧前, rGO 形貌较为柔顺; 而㷽烧后, 石墨

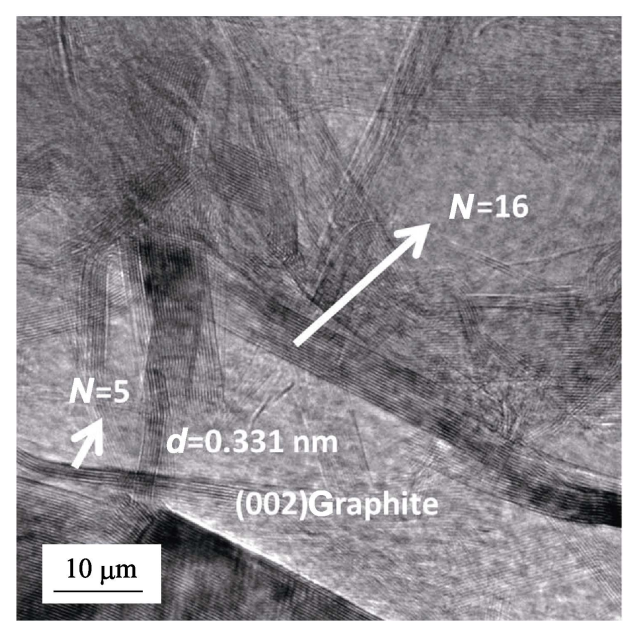

图 $1 \mathrm{rGO}$ 的高分辨 TEM 照片

Fig. 1 HRTEM image of rGO 
烯纳米片层棱角更为明显, 这是由于高温下 rGO 进 一步石墨化, 结晶度提高导致的。由此推断, 高温煅 烧可以提升 rGO 的力学性能。

本研究所采用 rGO 的 Raman 图谱如图 3(a)所 示, 其中 2 个主峰分别对应石墨烯的缺陷 D 峰 $\left(1335 \mathrm{~cm}^{-1}\right)$ 和面内振动 $\mathrm{G}$ 峰 $\left(1580 \mathrm{~cm}^{-1}\right)$ 。 $\mathrm{D}$ 峰反映 二阶双共振拉曼散射过程, 涉及到一个 iTO 声子与 一个缺陷的谷间散射; G 峰是一阶拉曼散射过程, 源自 $\mathrm{sp}^{2}$ 碳原子的面内振动, 是与布里渊区中心双 重简并的 iTO 和 iLO 光学声子相互作用产生的, 具 有 $\mathrm{E}_{2 \mathrm{~g}}$ 对称性。从图 3(b)中可以看出, 石墨烯混合碳 化硅粉体还出现了位于 772 和 $943 \mathrm{~cm}^{-1}$ 处的两个拉 曼峰, 这两个峰分别对应 $\mathrm{SiC}$ 的横光学(TO)和纵光 学(LO)特征振动。由于掺入的 rGO 含量较少, 相比 $\mathrm{SiC}$ 的拉曼峰, 石墨烯的 $\mathrm{D}$ 峰和 $\mathrm{G}$ 峰强度较弱。

\section{$2.2 \mathrm{rGO} / \mathrm{SiC}$ 复合材料密度及微观结构}

经过高温热压烧结, 不同 rGO 含量 $\mathrm{rGO} / \mathrm{SiC}$ 复 合材料的断面 SEM 照片如图 4 所示。随着 rGO 加 入量的增加, 嵌入碳化硅晶粒之间的条状材料等比 例增加, 其长度在 $8 \mu \mathrm{m}$ 左右, 与 $\mathrm{rGO}$ 的面向尺寸相 当, 由此推断条状材料为 $\mathrm{rGO}$ 纳米片。分散在 $\mathrm{rGO} / \mathrm{SiC}$ 复合材料中的黑点为炭黑, 主相为 $\mathrm{SiC}$ 晶
粒。材料断裂方式以穿晶断裂为主，通过断面较难 得到晶粒尺寸。从图 4(b) (d)中可以发现, $\mathrm{SiC}$ 晶粒 的生长被 $\mathrm{rGO}$ 纳米片阻隔。因此, 加入 rGO 后, 碳 化硅晶粒生长的路径被隔绝, 晶粒生长得到抑制。 但是，碳化硅陶瓷的致密化过程却没有被限制，图 4 中的 $\mathrm{rGO} / \mathrm{SiC}$ 复合材料均较为致密, $\mathrm{SiC}$ 晶粒内不存 在气孔。此外, 图 4 中 rGO 呈现定向平行排列的趋 势, 局部具有 zig-zag 结构, 总体呈现一定方向性。

这是由于热压过程中的压力作用使 $\mathrm{rGO}$ 纳米片 垂直于压力方向。烧结后 rGO 纳米片的面向尺寸与 加入时的面向尺寸都在 $8 \mu \mathrm{m}$ 左右(见图 2), 说明在 整个碳化硅烧结过程, rGO 并未发生分解开裂等现 象。An 等 ${ }^{[17]}$ 指出, 这种具有定向层状的石墨烯纳米 片掺杂结构有利于增加复合材料的力学性能。而这 种 zig-zag 结构也能抑制裂纹扩展和界面滑移, 增强 材料的断裂韧性 ${ }^{[18]}$ 。

所制备的 $\mathrm{rGO} / \mathrm{SiC}$ 复合材料的密度如图 5 所示, 随着 rGO 加入量的增加, 复合材料的相对密度有所 提升, 加入 $4 \mathrm{wt} \% \mathrm{rGO}$, 复合材料的相对密度达到最 高值, 为 $99.8 \%$, 实际密度达 $3.103 \mathrm{~g} / \mathrm{cm}^{3}$; 进一步增 加 $\mathrm{rGO}$ 加入量后，复合材料的相对密度下降为 $97.4 \%$ 。这可能与 rGO 的分散不均匀有关。图 6 是
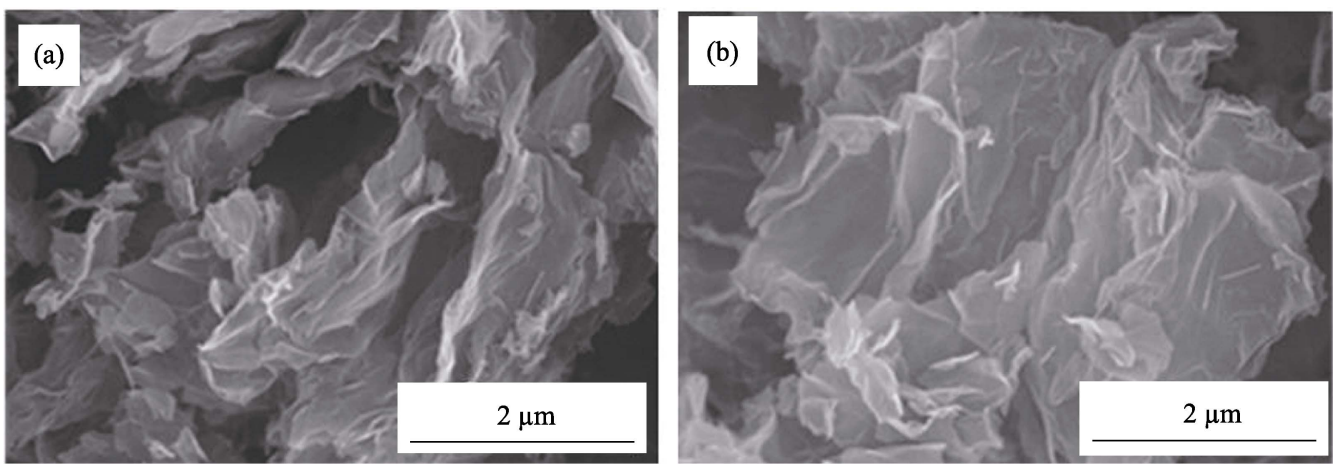

图 2 高温炦烧前(a)和后(b)rGO SEM 照片

Fig. 2 SEM images of rGO before (a) and after (b) calcination at $2050^{\circ} \mathrm{C}$
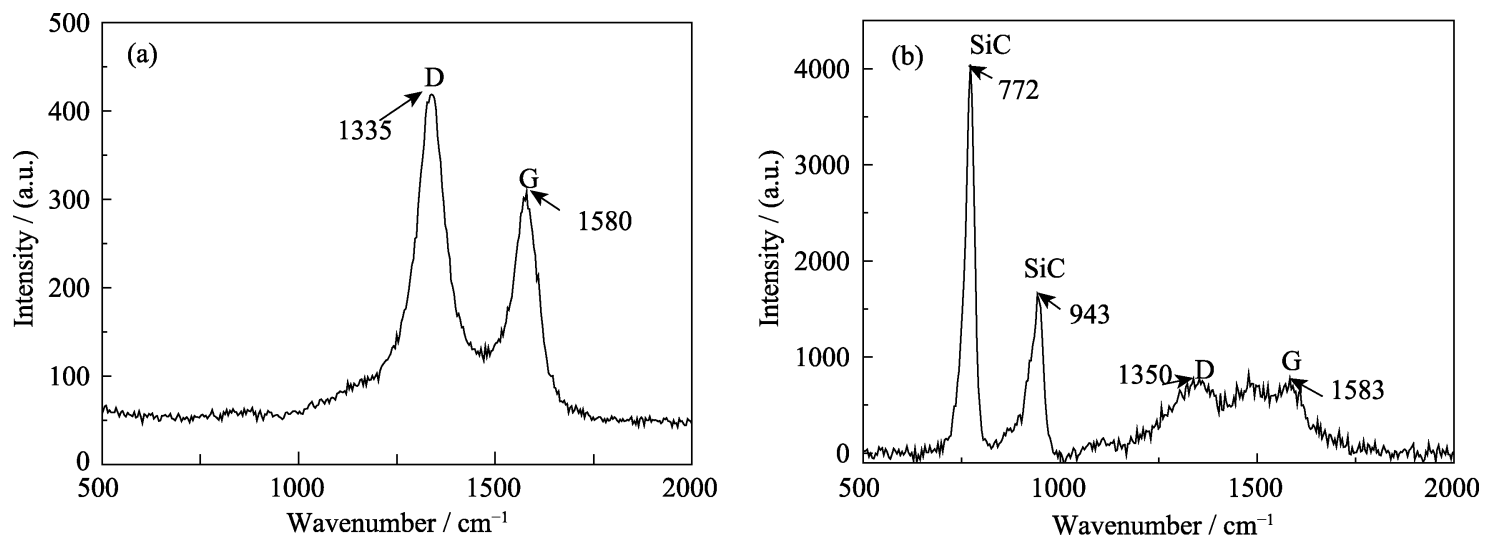

图 $3 \mathrm{rGO}(\mathrm{a})$ 和 $\mathrm{rGO}$ 混合 $\mathrm{SiC}$ 粉体(b)的拉曼图谱

Fig. 3 Raman spectra of rGO (a) and rGO/SiC mixture powder (b) 


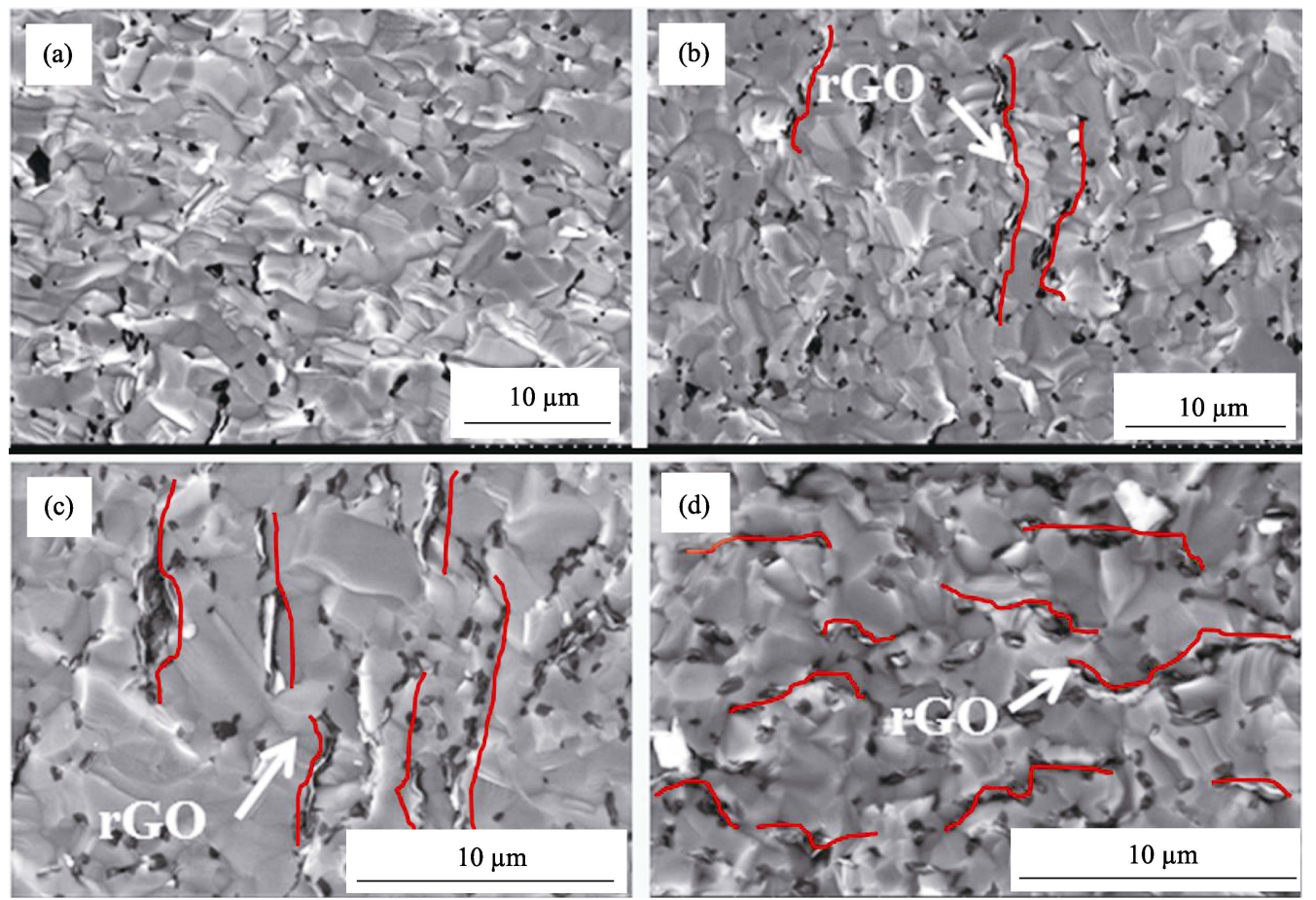

图 4 不同 rGO 含量复合材料的断面 SEM 照片

Fig. 4 SEM images of fractured surfaces of composites with different rGO contents (a) 0 ; (b) $2 \mathrm{wt} \%$; (c) $4 \mathrm{wt} \%$; (d) $6 \mathrm{wt} \%$

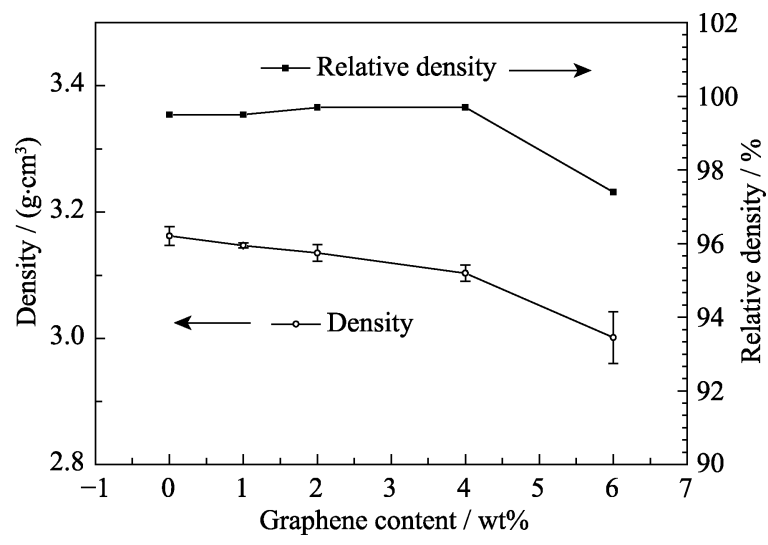

图 $5 \mathrm{rGO} / \mathrm{SiC}$ 复合材料密度与相对密度随 $\mathrm{rGO}$ 含量的变化 Fig. 5 Density and relative density of $\mathrm{rGO} / \mathrm{SiC}$ composites with different rGO contents
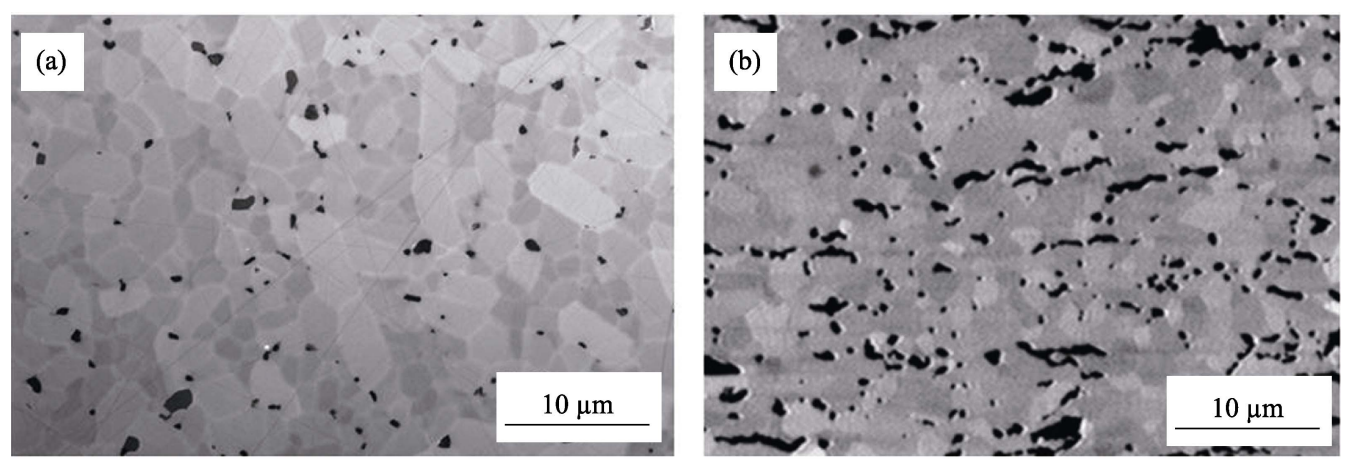

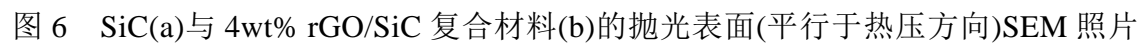

Fig. 6 SEM images of the polished surface (parallel to pressing direction) of $\mathrm{SiC}$ (a) and $4 \mathrm{wt} \% \mathrm{rGO} / \mathrm{SiC}$ composite (b)
$\mathrm{rGO} / \mathrm{SiC}$ 复合材料的抛光面微观形貌，抛光面平行 于热压方向。当 rGO 加入量为 0 时，如图 6(a)所示, 有部分炭黑颗粒分布在 $\mathrm{SiC}$ 晶界处; 由于通道祄度 的作用, $\mathrm{SiC}$ 晶粒清晰可见，部分晶粒有长柱状生长 趋势。当 rGO 加入量为 $4 \mathrm{wt} \%$ 时, 如图 6(b)所示, $\mathrm{SiC}$ 晶粒主要为等轴状; rGO 呈现平行条状排列的趋势。 由于 $\mathrm{rGO}$ 的加入, $\mathrm{SiC}$ 晶粒生长得到一定抑制, 晶粒 形貌由长柱状变为等轴状, 晶粒尺寸见表 1 。

随着 rGO 加入量从 $1 w t \%$ 增加到 $6 w t \%, S i C$ 晶 粒尺寸从 $2.02 \mu \mathrm{m}$ 略降至 $1.87 \mu \mathrm{m}$ 。考虑到测量误差 以及晶粒尺寸分布重叠等因素, rGO 对于晶粒的抑 制作用相当有限。这一尺寸与 Hanzel 等 ${ }^{[19]}$ 采用液相 烧结的方法以及 Belmonte 等 ${ }^{[13]}$ 采用 SPS 方法制 
表 1 不同 rGO 加入量下 rGO/SiC 复合材料中 $\mathrm{SiC}$ 的晶粒尺寸 $/ \mu \mathrm{m}$

Table 1 Grain size of $\mathrm{rGO} / \mathrm{SiC}$ composites with different $\mathrm{rGO}$ contents $/ \mu \mathrm{m}$

\begin{tabular}{ccccc}
\hline $\begin{array}{c}\mathrm{SiC}+ \\
\text { raGO }\end{array}$ & $\begin{array}{c}\mathrm{SiC}+ \\
\text { 1wt\%rGO }\end{array}$ & $\begin{array}{c}\mathrm{SiC}+ \\
\text { 2wt\%rGO }\end{array}$ & $\begin{array}{c}\mathrm{SiC}+ \\
\text { 4wt\%rGO }\end{array}$ & $\begin{array}{c}\mathrm{SiC}+ \\
6 \mathrm{wt} \% \mathrm{rGO}\end{array}$ \\
\hline $2.04 \pm 0.75$ & $2.02 \pm 0.65$ & $2.01 \pm 0.86$ & $1.86 \pm 0.58$ & $1.87 \pm 0.75$ \\
\hline
\end{tabular}

备的石墨烯纳米片/SiC 复合材料的晶粒尺寸相当。 与 Richard 等 ${ }^{[16]}$ 的工作相比, 晶粒生长得到很大抑 制, 他们采用石墨烯纳米片作为烧结助剂, 而不加 炭黑, 晶粒尺寸达到 $10 \mu \mathrm{m}$, 说明相比石墨烯纳米 片，少量炭黑抑制晶粒生长的效果更好。

为了进一步研究 $\mathrm{rGO}$ 定向掺杂 $\mathrm{SiC}$ 复合材料的 定向度和真实性, 通过能谱对其微观形貌做元素线 扫描, 如图 6 所示。从扫描结果看, 材料中只发现 C、Si 两种元素; C 元素分布呈现间隔出峰的状态, 遇片状结构出高峰, 遇颗粒状结构则平缓; Si 元素 的分布与之相反, 遇片状结构出现谷峰, 遇颗粒状 结构则表现平稳。由此, 基本可以确定, 颗粒状结构 就是加入的碳化硅, 而带状结构应是加入的石墨烯
的侧面, 并且 rGO 的掺杂呈现出定向的特点。

\section{$2.3 \mathrm{rGO} / \mathrm{SiC}$ 复合材料的力学性能及其增韧 机理}

$\mathrm{rGO} / \mathrm{SiC}$ 复合材料的力学性能如图 8 所示。掺 入 $1 \mathrm{wt} \% \mathrm{rGO}$ 后，复合材料的抗弯强度相比不掺石 墨烯的热压 SiC 陶瓷有所下降。但是, 随着掺入量 的增加, 复合材料的抗弯强度逐渐提高, 掺入 $4 \mathrm{wt} \%$ 时, 抗弯强度达到最高值, 为 $564 \mathrm{MPa}$, 比热压碳化 硅陶瓷提高了 $6 \%$; 断裂韧性为 $4.02 \mathrm{MPa} \cdot \mathrm{m}^{1 / 2}$, 比热 压碳化硅陶瓷提高了 $54 \%$ 。当 $\mathrm{rGO}$ 掺入量为 $6 \mathrm{wt} \%$ 时, 复合材料的抗弯强度下降至 $420 \mathrm{MPa}$, 低于热 压碳化硅陶瓷; 但是复合材料的断裂韧性达到 $4.56 \mathrm{MPa} \cdot \mathrm{m}^{1 / 2}$, 比热压碳化硅陶瓷提高了 $75 \%$ 。rGO 掺入量为 $6 \mathrm{wt} \%$ 的复合材料的相对密度只有 $97.4 \%$, 但是其力学性能依然较高, 这主要归功于 rGO 优异 特性。Richard 等 ${ }^{[16]}$ 也采用的是热压方法, 与其相比, 本研究制备的复合材料的抗弯强度是其 2 倍以上, 断裂㓞性相当。这一结果还优于无压烧结得到的 $\mathrm{rGO} / \mathrm{SiC}$ 复合材料 ${ }^{[15]}$ 。

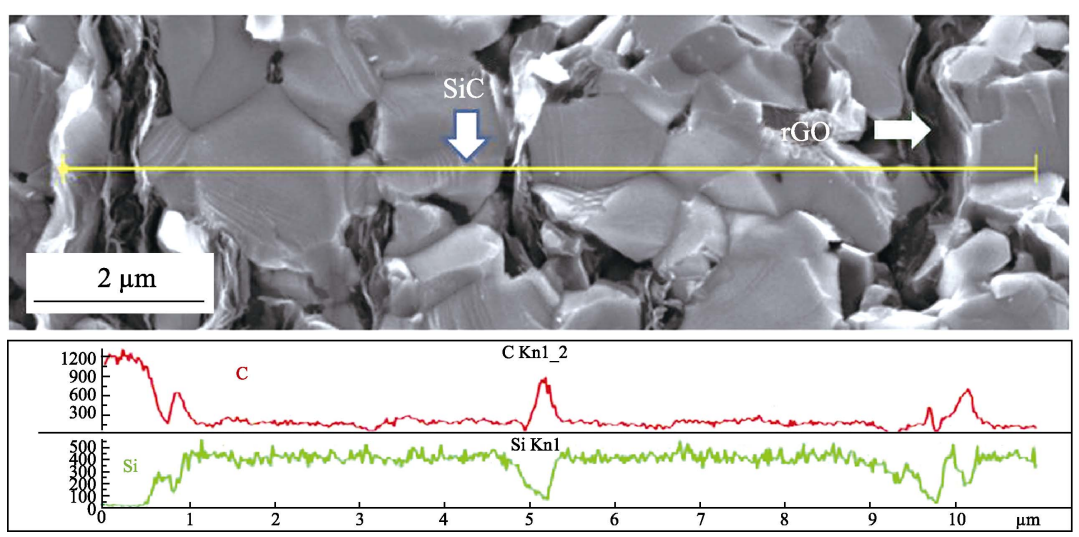

图 7 石墨烯定向掺杂碳化硅陶瓷的元素微观线扫描分析

Fig. 7 Elemental lining scanning of SiC ceramics with rGO oriental doping

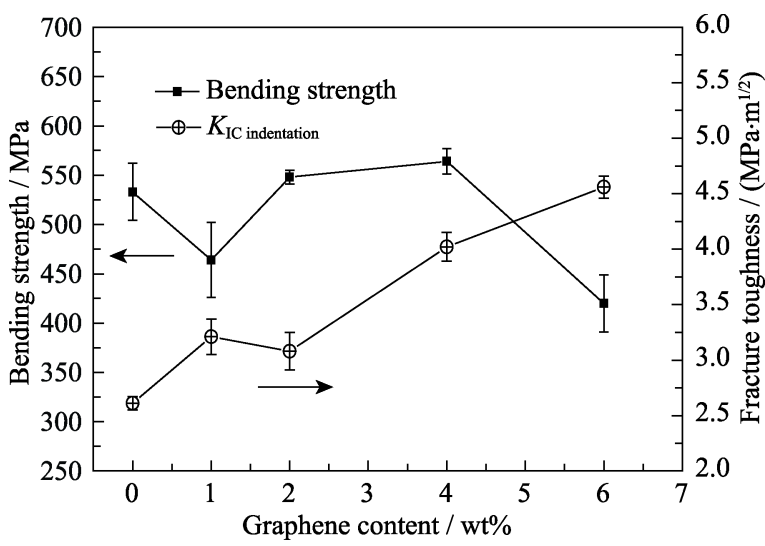

图 $8 \mathrm{rGO} / \mathrm{SiC}$ 复合材料的抗弯强度与断裂韧性随 $\mathrm{rGO}$ 加入 量的变化

Fig. 8 Bending strength and fracture toughness of $\mathrm{rGO} / \mathrm{SiC}$ composites with different rGO contents
为了进一步研究 $\mathrm{rGO}$ 掺杂对 $\mathrm{rGO} / \mathrm{SiC}$ 复合材料 力学性能的提升作用, 采用 TEM 观察了复合材料 减薄片的断裂形貌(如图 9 所示)。TEM 照片显示，微 观上石墨烯纳米片能很好地复合在碳化硅晶粒的断 口处。片状石墨烯纳米片有拉伸和撕裂的痕迹，整 体呈破裂状态，说明在复合材料断裂过程中出现了 明显的 rGO 拔出的现象, 进一步证实复合材料力学 性能的提高与添加 rGO 有关。 $\mathrm{Liu}$ 等 ${ }^{[20]}$ 认为, 拔出 石墨烯纳米片比拔出纳米纤维需要更多的能量。另 外, 在致密化过程中石墨烯纳米片顺着晶粒生长出 现的 zig-zag 结构, 进一步增大了石墨烯与晶粒的接 触面积，从而有效起到增韧作用。

$\mathrm{rGO} / \mathrm{SiC}$ 复合材料的裂纹扩展情况如图 10 所 


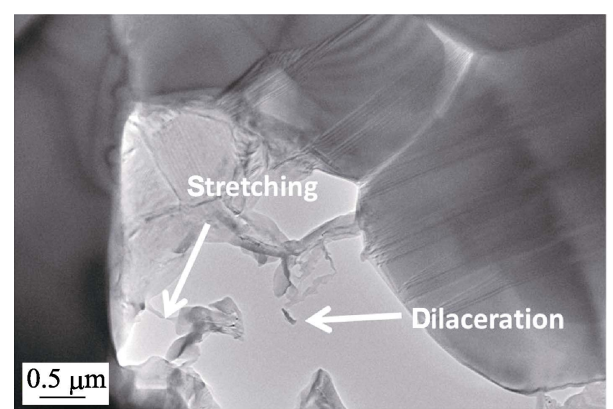

图 $9 \mathrm{rGO} / \mathrm{SiC}$ 复合材料减薄片的断裂 $\mathrm{TEM}$ 照片

Fig. 9 TEM image of fracture face of $\mathrm{rGO} / \mathrm{SiC}$ composite
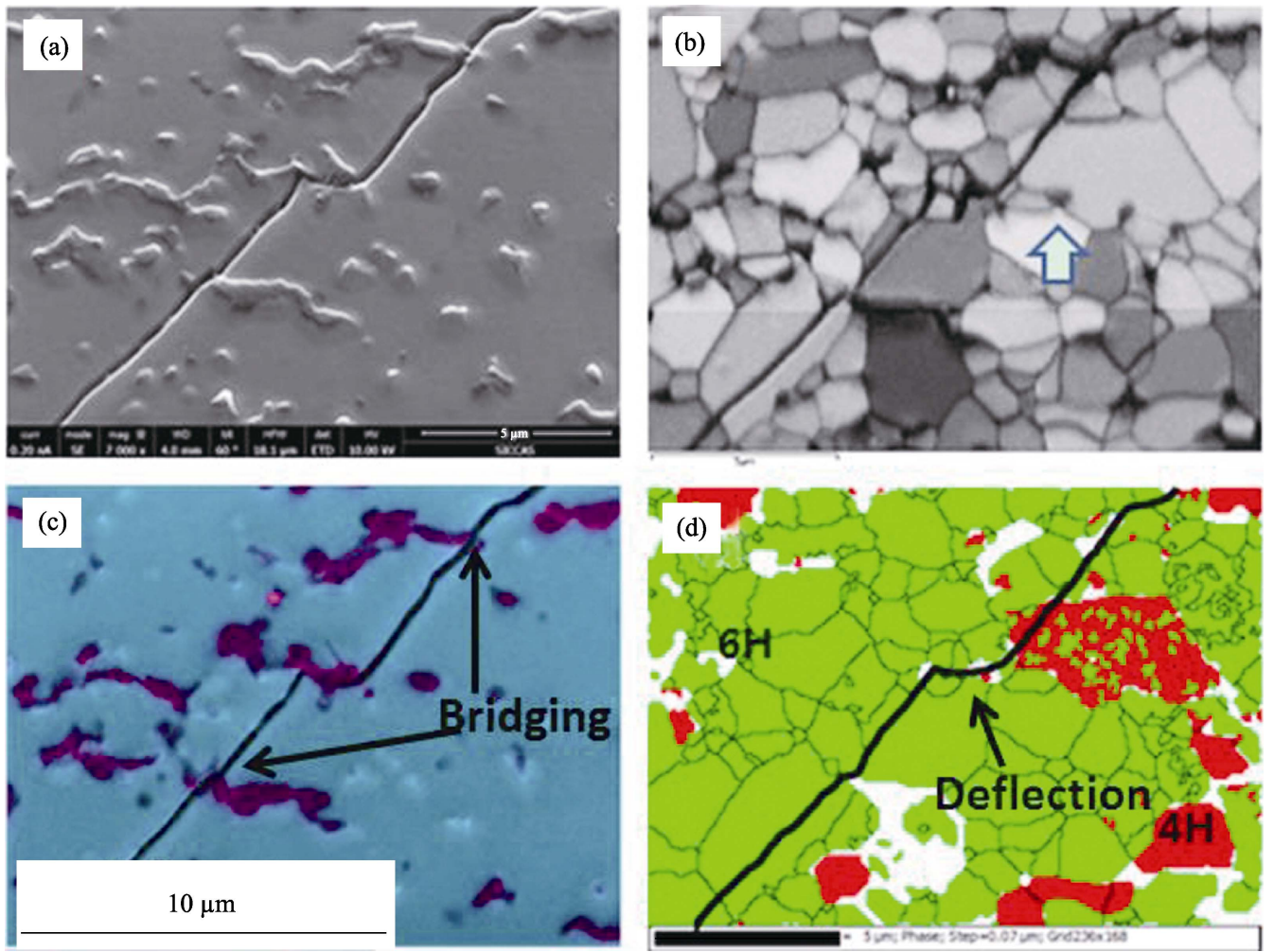

图 $10 \mathrm{rGO} / \mathrm{SiC}$ 复合材料的增韧机理

Fig. 10 Toughening mechanism of rGO/SiC composite

\section{3 结论}

通过热压烧结制备了 $\mathrm{rGO}$ 定向掺杂的 $\mathrm{rGO} / \mathrm{SiC}$ 复合材料, 通过对复合材料中 rGO 的掺入量、微观 结构和力学性能相互关系进行分析与讨论, 得到如 下结论:

1) 复合材料的断裂方式以穿晶断裂为主。

2) 加入 rGO 后, 复合材料的断裂㓞性得到明 显提高。加入 $4 \mathrm{wt} \% \mathrm{rGO}$ 时, 复合材料的抗弯强度 达到最高值 $564 \mathrm{MPa}$ ，比热压碳化硅陶瓷提高了 6\%; 断裂韧性达到 $4.02 \mathrm{MPa} \cdot \mathrm{m}^{1 / 2}$, 比热压碳化硅陶瓷提 高了 $54 \%$ 。

3）当 rGO 加入量为 $6 \mathrm{wt} \%$, 复合材料的断裂韧
示。复合材料的断裂方式主要为穿晶断裂，见图 10(b)。 裂纹按照其扩展方向直接穿过晶粒, 而不是沿着晶 界进行扩展。但是, 由于 rGO 的存在, 裂纹走向发 生了偏转和桥连现象, 见图 10(c)。复合材料中 SiC 晶粒包括 $6 \mathrm{H}$ 相(绿色)和 $4 \mathrm{H}$ 相(红色), 其中 $6 \mathrm{H}$ 相 为主相, 见图 10(d)。因此, 结合图 9 中对石墨烯纳 米片拔出现象的观察, 可以认为 $\mathrm{rGO}$ 的拔出、裂纹 偏转和桥连是 $\mathrm{rGO} / \mathrm{SiC}$ 复合材料㓞性提高的主要 原因。 
mobility in suspended graphene. Solid State Commun., 2008, 146(9/10): 351-355.

[5] JIANG D, ZHANG J. Properties of Carbide Ceramics from Gelcasting and Pressure-less Sintering. 3rd International Congress on Ceramics. IOP Conference Series-Materials Science and Engineering, 2011, 182011.

[6] AHMAD SERJOUEI G G, ZHANG X F, SRIDHAR IDAPALAPATI, et al. On improving ballistic limit of bi-layer ceramic-metal armor. International Journal of Impact Engineering, 2017, 105(SI): 54-67.

[7] LUO H Y, CHEN W N W, RAJENDRAN A M. Dynamic compressive response of damaged and interlocked SiC-N ceramics. Journal of the American Ceramic Society, 2006, 89(1): 266-273.

[8] VIROJANADARA C, SYJARVI M, YAKIMOVA R, et al. Homogeneous large-area graphene layer growth on $6 \mathrm{H}-\mathrm{SiC}$ (0001). Phys. Rev. B, 2008, 78(24): 245403-1-6.

[9] LIORENTE J, ROMAN MANSO B, MIRANZO P, et al. Tribological performance under dry sliding conditions of graphene/ silicon carbide composites. Journal of the European Ceramic Society, 2016, 36(3): 429-435.

[10] Li Q S, ZHANG Y J, GONG H Y, et al. Effects of graphene on the thermal conductivity of pressureless-sintered SiC ceramics. Ceramics International, 2015, 41(10): 13547-13552.

[11] ROMAN-MANSO B, FIGUEIREDO F M, ACHIAGA B, et al. Electrically functional 3D-architectured graphene/SiC composites. Carbon, 2016, 100: 318-328.

[12] ASL M S, KAKROUDI M G. Characterization of hot-pressed graphene reinforced $\mathrm{ZrB}_{2}$-SiC composite. Materials Science and En- gineering a-Structural Materials Properties Microstructure and Processing, 2015, 625: 385-392.

[13] BELMONTE M, NISTAL A, BOUTBIEN P, et al. Toughened and strengthened silicon carbide ceramics by adding graphene-based fillers. Scripta Materialia, 2016, 113: 127-130.

[14] MIRANZO P, RAMIREZ C, ROMAN-MANSO B, et al. In situ processing of electrically conducting graphene/SiC nanocomposites. Journal of the European Ceramic Society, 2013, 33(10): 1665-1674.

[15] LI Q S, ZHAND Y J, GONG H Y, et al. Enhanced fracture toughness of pressureless-sintered SiC ceramics by addition of graphene. J. Mater. Sci. Technol., 2016, 32(7): 633-638.

[16] SEDLAK R, KOVALCIKOVA A, GIRMAN V, et al. Fracture characteristics of $\mathrm{SiC} /$ graphene platelet composites. Journal of the European Ceramic Society, 2017, 37(14): 4307-4314.

[17] AN Y M, HAN J C, ZHANG X H, et al. Bioinspired high toughness graphene $/ \mathrm{ZrB}_{2}$ hybrid composites with hierarchical architectures spanning several length scales. Carbon, 2016, 107: 209-216.

[18] LIU L X, WANG Y, LI X H, et al. Enhancing toughness in boron carbide with reduced graphene oxide. Journal of the American Ceramic Society, 2016, 99(1): 257-264.

[19] HANZEL O, SEDLAK R, SEDLACEK J, et al. Anisotropy of functional properties of $\mathrm{SiC}$ composites with GNPs, GO and in-situ formed graphene. Journal of the European Ceramic Society, 2017, 37(12): 3731-3739.

[20] LIU J, YAN H X, REECE M J, et al. Toughening of zirconia/alumina composites by the addition of graphene platelets. Journal of the European Ceramic Society, 2012, 32(16): 4185-4193. 